\title{
COMPARING YIQ AND YCBCR COLOUR IMAGE TRANSFORMS FOR SEMI-FRAGILE MEDICAL IMAGE STEGANOGRAPHY
}

\author{
${ }^{a, b}$ Peter Eze, ${ }^{a}$ Udaya Parampalli, ${ }^{a}$ Robin Evans and ${ }^{b}$ Dongxi Liu \\ ${ }^{\mathrm{a}}$ University of Melbourne, ${ }^{\mathrm{b}}$ CSIRO Data61, Australia
}

\begin{abstract}
Visible light photography diagnostic images are coloured $e x$ vivo medical images popularly used in Dermatology and Endoscopy for diagnosis and monitoring. The need to protect the integrity of these images as well as associated patient data calls for techniques such as image steganography and watermarking. This research explores and compares the effect of watermarking on the YIQ and $\mathrm{YCbCr}$ colour transforms used in processing digital coloured images and video in recent times. Using a new spread spectrum watermarking algorithm, it was found that YIQ has better distortion performance than $\mathrm{YCbCr}$ in the order of $\mathbf{3 d \boldsymbol { B }}$ while $\mathrm{YCbCr}$ had lower BER for accurate watermark retrieval and tamper detection in the order of $1.3 \times 10^{-3}$.
\end{abstract}

Index Terms - Colour Model, Steganography, Medical Image, C4S, distortion

\section{INTRODUCTION}

Visible light photography is one of the important types of Diagnostic medical images apart from radiological, Printed Signal, Microscopy and 3D-Recostruction diagnostic images. They are often used for ex vivo imaging and the rays used are in the visible region of the electromagnetic spectrum. They are popularly used in Dermatology, Endoscopy and Ophthalmology [1]. Unlike X-ray, MRI, CT, and Ultrasound scans that are mostly produced in vivo; are gray-scale and stored in DICOM formats, visible light medical images are mostly created from outside the body; has variety of colours and could be stored in normal image formats such as JPEG, gif, tiff or PNG.

Coloured images, as opposed to grayscale images, contain not only intensity information but also colour information such as chrominance. Unlike grayscale images that are made up of just one channel, coloured images are made up of three channels: Red, Green and Blue [2]. True colour images use 8 bits to represent each of the colour channels.

In digital signal processing, coloured images are not often processed directly in the RGB colour space. They are either converted to grayscale (if it is appropriate for the application) or into another colour model before further processing occurs. This is because the RGB colour model is device-dependent and the actual values depend on the device in which the colours were captured or displayed $[3,4,5]$. The reasons for choosing a different colour space other than RGB include: computing device-independent values which could be used for computations without reference to a specific device; concentration of signal energy into few components thereby aiding compression; decorrelation of transform components to aid independent manipulation of variables and robustness while source statistics changes [5].

The importance of determining accurate representation cannot be overemphasized in medicine. As automated healthcare systems evolve and most decisions are made by machines, it is important that data representation in the machines are accurate representation of the actual symptoms and biomarkers captured from the body. This will ensure that predictions, monitoring and diagnostic outcomes are as accurate as possible.

The objective and contribution of this work include: (i) evaluating the YIQ and $\mathrm{YCbCr}$ colour models for tamper detection in coloured medical images while using a variant of Spread Spectrum $\left(\mathrm{C}_{4} \mathrm{~S}\right)$ Steganography [17], (ii) evaluating the localised rather than global image distortion of adaptive embedding strength for the Spread Spectrum Steganography for tamper detection, (iii) studying the conflict between JPEG image compression and effective tamper detection in coloured medical images.

Section 2 provides background while section 3 describes related works. Sections 4 and 5 describes the dataset and experimental procedure respectively. Section 6 presents results and discussions before the work concludes in section 7 while providing directions for future work.

\section{COLOUR TRANSFORMS}

Transform solutions define a new coordinate system or basis that can remove sample dependence and enable scalar quantisation and independent sample handling. The new basis, when well chosen, can lead to more efficient coding by providing lower distortion at same information rate and vice versa.

To the human visual system (HVS), coloured images are represented by the intensity function, $I\left(x^{\prime}, y^{\prime}, \lambda\right)$ where $x$ and $\mathrm{y}$ parameters relate to its current position and the wavelength, $\lambda$, is the wavelength of the incident light. The wavelength of visible light in the Electromagnetic Spectrum 
ranges from $380 \mathrm{~nm}$ to $780 \mathrm{~nm}$ [4]. Achromatic light or grayscale colours are defined only by intensity while chromatic light contains colours and are defined by three main properties: Radiance, Luminance and Brightness. Luminance is a spectrum of gray levels.

For a spectral radiance of visible light, $\boldsymbol{L}$, consisting of wavelength, $\boldsymbol{\lambda}$, the values of $\boldsymbol{X}, \boldsymbol{Y}$ and $\boldsymbol{Z}$ are given as:

$$
\begin{aligned}
& X=\int_{380}^{780} L(\lambda) \mathrm{x}^{\prime}(\lambda) \mathrm{d} \lambda \\
& Y=\int_{380}^{780} L(\lambda) \mathrm{y}^{\prime}(\lambda) \mathrm{d} \lambda \\
& Z=\int_{380}^{780} L(\lambda) \mathrm{z}^{\prime}(\lambda) \mathrm{d} \lambda
\end{aligned}
$$

The components $x^{\prime}, y^{\prime}$ and $z^{\prime}$ are mainly parts of the incident wavelength that corresponds to blue, green and red lights respectively. The cones of the eyes (retina precisely), which detects chromatic lights are divided into three groups: short, middle and long. These cones are sensitive to the corresponding parts of the visible light, namely: blue, green and red, respectively.

From the tri-stimulus values $\mathrm{X}, \mathrm{Y}$ and $\mathrm{Z}$, different chromaticity coordinates could be derived. These form the basis of different colour models which serves different purposes among which are: RGB, CMYK; HSI, HSV, HSL; YIQ, YUV, YCbCr and CIEXYZ, CIELAB. In this research we are concerned with the YIQ and YCbCr colour spaces which are popularly used for colour compression in digital video and TV coding and transmission.

$Y I Q$ and $\mathbf{Y C b C r}$ are the most popular transforms that helps one extract the Intensity (Luma) and the corresponding chromaticity which describe the colour details in each of the channels. In both models, $\boldsymbol{Y}$ is a luminance signal coded in 8 bits and it approximately represents the grayscale equivalent of a colour image [5].

YIQ relates to analogue videos while $\mathrm{YCbCr}$ are commonly used for coding digital video, JPEG and MPEG formats. $\mathbf{I}$ is an in-phase component in the Orange-Cyan axis while $\mathbf{Q}$ is quadrature component along the MagentaGreen axis. The sensitivity of the HVS to luminance than to hue and saturation is the major design principle behind the YIQ basis colour transform.

Both $\mathrm{Cr}$ and $\mathrm{Cb}$ in $\mathrm{YCbCr}$ represent chrominance values which relate to colour details of the digital image. All transformations originate from the CIE XYZ abstract base transform representing an independent observer.

In the case of colour transforms, however, each RGB pixel gets transform into other equivalent values in the new basis. For all transforms, R, G and B values must be converted into the domain $[0,1]$ using appropriate scaling factors. For N-bit colour per channel, the scaling factor is $1 / 2^{\mathrm{N}}-1$. For 8-bit per channel true colour images, for example, the scaling factor is $1 / 255$.
The conversion from $\boldsymbol{R} \boldsymbol{G B}$ to $\boldsymbol{Y I Q}$ is based on the transformation:

$$
\left[\begin{array}{l}
Y \\
I \\
Q
\end{array}\right]=\left[\begin{array}{ccc}
0.299 & 0.587 & 0.114 \\
0.596 & -0.274 & -0.322 \\
0.211 & -0.522 & 0.311
\end{array}\right]\left[\begin{array}{l}
R \\
G \\
B
\end{array}\right]
$$

The inverse transform from $Y I Q$ to $R G B$ is:

$$
\left[\begin{array}{l}
R \\
G \\
B
\end{array}\right]=\left[\begin{array}{ccc}
1 & 0.956 & 0.623 \\
1 & -0.272 & -0.648 \\
1 & -1.105 & 0.705
\end{array}\right]\left[\begin{array}{l}
Y \\
I \\
Q
\end{array}\right]
$$

For conversion between RGB and $\mathrm{YCbCr}$, if the input is uint8, $\boldsymbol{Y}$, is in the range $[16,235]$, and $\mathrm{Cb}$ and $\mathrm{Cr}$, are in the range $[16,240] . \boldsymbol{Y}$ is luminance (luma), $\boldsymbol{C b}$ is blue minus luma (B - Y) while $\boldsymbol{C r}$ is red minus luma (R - Y).

The transform from $\boldsymbol{R G B}$ to $\mathbf{Y C b C r}$ is:

$\left[\begin{array}{c}Y \\ C b \\ C r\end{array}\right]=\left[\begin{array}{c}16 \\ 128 \\ 128\end{array}\right]+\frac{1}{255}\left[\begin{array}{ccc}65.481 & 128.553 & 24.966 \\ -37.797 & -74.203 & 112 \\ 112 & -93.786 & -18.214\end{array}\right]\left[\begin{array}{l}R \\ G \\ B\end{array}\right]$

The inverse transform back to $\boldsymbol{R} \boldsymbol{G B}$ space is given by equation

$\left[\begin{array}{l}R \\ G \\ B\end{array}\right]=\frac{1}{255}\left[\begin{array}{ccc}0.00456821 & 0.0 & 0.00625893 \\ 0.00456621 & -0.00153632 & -0.00318811 \\ 0.00456621 & 0.00791071 & 0.0\end{array}\right]\left(\left[\begin{array}{c}Y \\ C b \\ C r\end{array}\right]-\left[\begin{array}{c}16 \\ 128 \\ 128\end{array}\right]\right)$

These colour spaces will be used for watermarking and compared in terms of Peak Signal-to-noise ratio (PSNR), Structural Similarity Index Measure (SSIM) and Bit Error Rate (BER) in this research.

\section{RELATED WORKS}

The use of various image colour transforms including HSV, YIQ, YUV and $\mathrm{YCbCr}$ has been experimented upon by some researchers $[6,7,8,9]$ for image representation and computational purposes including watermarking and steganography. However, most of the researchers had used each of the colour spaces and associated image transforms in isolation and only few have tried to compare the outcome of each colour space in the domain of digital steganography.

Sejpal and Shah in [6] carried out a performance analysis of Lifting Wave Transform (LWT) and Discrete Wavelet Transform watermarking on the colour models of YIQ, YUV and YCbCr while comparing the PSNR performance of each of the channels in the colour models. However, the comparison were done for image watermark and for robust image watermarking. Due to several transforms implemented (LWT and DWT) it was not clear if a comparison is being made for the colour spaces or for the transforms. However, the authors recommended Y-channel and LWT watermark method combination for robust watermarking systems.

A robust watermarking for coloured images was performed by Mitrea et al in [9]. They used HSV colour space but did not compare with other colour models. However, the work was very closely related to Cox [10] and Ruanaidh [11] methods for Spread-Spectrum watermarking, which is related to our watermarking method in this research, but being adapted for semi-fragile watermarking 
and tamper detection instead of being strictly applied to robust watermarking.

\section{EVALUATION DATA SET}

The data set used was taken from an open image database: International Skin Imaging Collaboration (ISIC). Specifically, our data set was extracted from the "ISIC 2018: Skin Lesion Analysis Towards Melanoma Detection" grand challenge datasets [12][13]. The images to be watermarked consists of 1447 benign images and 363 malignant images out of the total 2594 images in the public data set. The unclassified images are either benign or malignant but were used as test images for the competition. A sample of malignant and benign images from the data set is shown in fig 1 below.

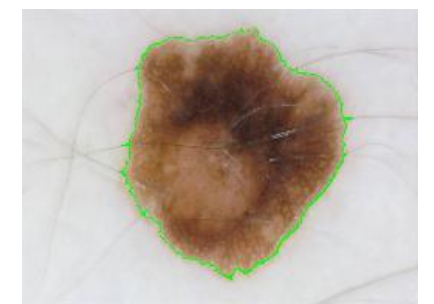

Fig 1 - ROI-Segmented Sample Data Set

\section{EXPERIMENTAL PROCEDURE}

The steps employed in carrying out this experiment include:

i. Load the original images from a folder, separating the benign and malignant lesions.

ii. For each image in each image class, divide it into $8 \times 8$ blocks.

iii. For each sub-block take YIQ transform.

iv. Using the distortion optimised version of $\mathrm{C}_{4} \mathrm{~S}$ [17] embedding algorithm and an augmented 63-bit gold code, $\boldsymbol{W}$, insert watermark bit into the $\mathrm{Y}$ Channel using equation (6) :

$Y_{i j}=\left\{\begin{array}{l}X_{i j}+\alpha W_{i j}, S_{a}=0 \\ X_{i j}-\alpha W_{i j}, S_{a}=1\end{array}\right.$

$\mathbf{X}=$ Original Channel, $\mathbf{Y}=$ Watermarked Channel

v. Convert the sub-block back into RGB domain.

vi. Compute PSNR, SSIM, HSI and record the dynamic embedding strength, $\boldsymbol{\alpha}$, for the subblock.

vii. Save the image back as .JPG with a quality factor of 100 into a different folder.

viii. Load the $\mathrm{C}_{4} \mathrm{~S}$ extraction algorithm and supply the corresponding parameters such as watermarked image folder, $\boldsymbol{W}$ and length of watermark.

ix. Extract the watermarks using (7) and measure BER.

$$
S^{(a)}=\left\{\begin{array}{l}
0,\langle Y, W\rangle=p \pm \varepsilon \\
1,\langle Y, W\rangle=-p \pm \varepsilon
\end{array}\right.
$$

$\mathbf{p}$ is pre-determined base correlation value (BCV).

$\mathrm{x}$. Computer $R X$ correlation.

xi. Plot the deviations in correlation at embedder $(\boldsymbol{T} \boldsymbol{X})$ and at the extractor $(\boldsymbol{R} \boldsymbol{X})$. xii. Repeat steps iv - xi for each of the I and Q channels.

xiii. Repeat iii - xi for YCbCr Colour model.

xiv. Repeat xii for each of $\mathrm{Cb}$ and $\mathrm{Cr}$ channels.

\section{RESULTS AND DISCUSSION}

The Results and discussions will be presented according to the three objectives of this work in order to provide direct answers and insights.

\subsection{YIQ and YCbCr Steganography Comparison}

Fig 2a shows that YIQ colour space provides up to $3 \mathrm{~dB}$ higher PSNR than $\mathrm{YCbCr}$ for all $\mathrm{BCV}$ values used in the experiment.

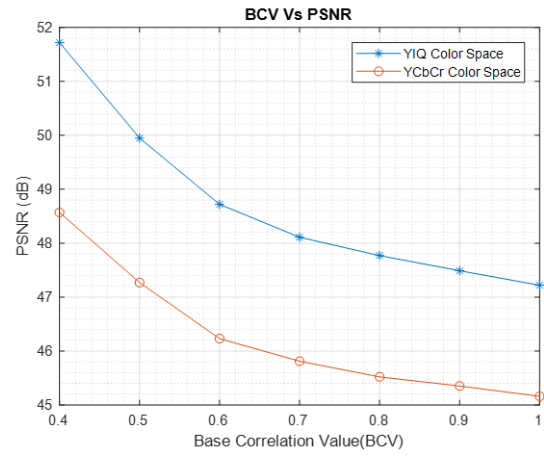

(a)PSNR

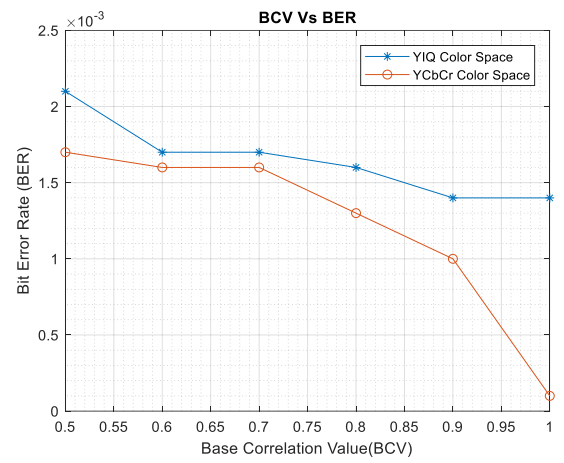

(b) BER

Fig 2 - Performances of YIQ and $\mathrm{YCbCr}$

Both YIQ and $\mathrm{YCrCb}$ have considerably good distortion (Fig 2a) and Error (Fig 2b) performance for watermarking in the Y-Channel. This is because both global and average localised PSNR are above the 40dB benchmark for medical images [14].

Further experiments were carried out for $\mathrm{YCbCr}$ as it has better BER performance with acceptable distortion (PSNR). Table 1 shows that $\mathrm{Cr}$ had best distortion performance but worst BER value.

Table 1: Result of watermarking each channel with $\mathrm{C}_{4} \mathrm{~S}$ CHANNEL

\begin{tabular}{ccc}
$\begin{array}{c}\text { AVG. } \\
\text { PSNR } \\
\text { (DB) }\end{array}$ & $\begin{array}{c}\text { AVG. } \\
\text { SSIM }\end{array}$ & BER \\
\hline 45.29 & 0.9959 & 0.0019 \\
45.68 & 0.9889 & 0.0610 \\
46.60 & 0.9959 & 0.0687 \\
\hline
\end{tabular}


Hence, the Y-Channels of both YIQ and YCbCr has good performance in both accuracy and distortion; however, the error rates are higher in their Chrominance channels even though better distortion performance may be obtained in their channels.

\subsection{Spread Spectrum localized Image Distortion}

Fig 3 compares the values of dynamically computed local embedding strength, $\boldsymbol{\alpha}$ with corresponding locally computed PSNR values, which is a measure of distortion. The quest into local distortion was raised in [15] with respect to Improved Spread Spectrum introduced in [16].

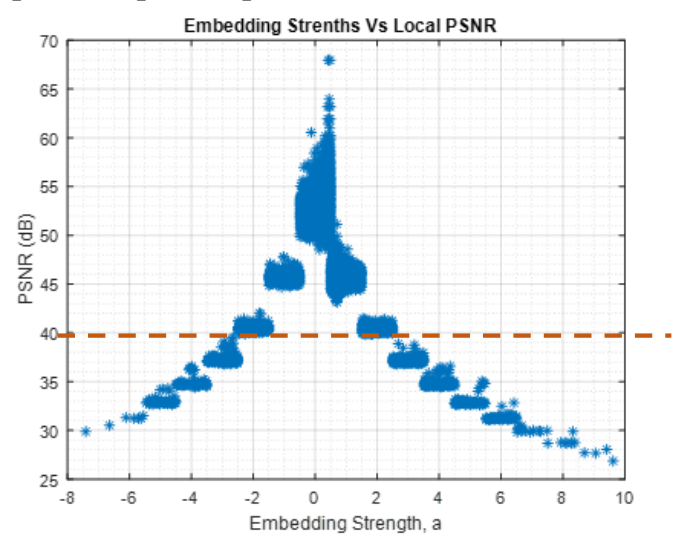

Fig 3 - Embedding Strength Vs Local Distortion

With 40dB threshold for local medical image blocks instead of the global image, what percentage of image blocks fell below this baseline? This was computed with the same data (81, 920 image sub-blocks) used to plot Fig 3. It was found out that, for the malignant lesion images, $2.46 \%$ fell below the $40 \mathrm{~dB}$ threshold while it is only $\mathbf{0 . 9 6 \%}$ for equivalent number of benign images. Though the percentage that fall within ROI specifically may need to be computed, this is a promising result. With larger datasets of about 2594 images consisting of both malignant and benign lesions, the average percentage of local sub-block distortions below $40 \mathrm{~dB}$ averaged to $\mathbf{1 . 0 7 \%}$. Hence, local distortions exist but are low and would not affect diagnosis.

\subsection{JPEG Compression and Tamper Detection}

The $\mathrm{Cb}$ and $\mathrm{Cr}$ channels of $\mathrm{YCbCr}$ colour model behave like high frequency channels and are easily affected by JPEG reconstruction and saving of images. Hence, their error tolerance is low and is not recommended for text embedding. Up to 687 bits for every 10,000 bits could be retrieved in error. This is opposed to Y-channel with only up 19 error bits per 10,000 bits extracted. Fig 4 shows how saving the watermarked image in JPEG has shifted the correlation value distributions from ' $\boldsymbol{T} \boldsymbol{X}$ ' to ' $\boldsymbol{R} \boldsymbol{X}$ ' values.

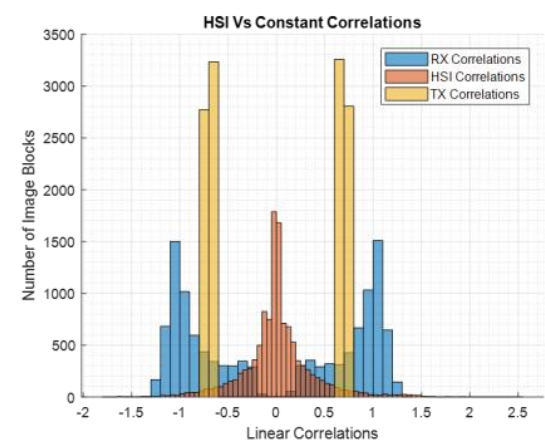

Fig 4 - Y-Channel watermarking and JPEG compression

Hence, compressed file formats are not desirable for Steganogaphic manipulation of images for semi-fragile tamper detection, as the compression mechanism is a form of allowed tampering provided diagnostic information is not affected. Other file formats would be explored in future.

\section{CONCLUSION}

The YIQ and YCbCr Colour spaces have been compared on their ability to preserve original image quality as well as allow the correct extraction of added bits representing medical records as well as tamper detection. It can be seen that YIQ embedding in the Y-Channel has better distortion performance while $\mathrm{YCbCr}$ has better watermark detection accuracy. Both transforms has considerably good distortion performance with the $\mathrm{C}_{4} \mathrm{~S}$ watermarking algorithm as all global PSNR values were above $40 \mathrm{~dB}$ threshold recommended for medical images [14]. Furthermore, as local image distortion needs to be considered in medical images especially for ROI, it has also been determined that up to $1.07 \%$ of local image sub-blocks could have distortion rates below $40 \mathrm{~dB}$. It is also worthy of note that the file format could affect the Steganographic performance of these colour spaces. JPEG formats and other compression-prone file formats has greater influence than tiff and other noncompressed file formats.

For further research, the percentage or exact number of blocks that lie within the region of interests (ROI) as defined in fig 1, which has local distortion below the acceptable threshold would be determined and further optimisation carried out to reduce this value to zero.

\section{ACKNOWLEGEMENT}

Research supported by CSIRO Data61 and Melbourne Research Scholarship (MRS) of the University of Melbourne Australia. 


\section{REFERENCES}

[1] F.E.A El-Gamal, M. Elmogy and A. "Current Trends in medical image registration and Fusion" In Egyptian Informatics Journal, 2015, http://dx.doi.org/10.1016/j.eij.2015.09.002.

[2] C. Saravanan "Color Image to Gray Image conversion" In $2^{\text {nd }}$ International Conference on computer Engineering Applications, pp. $196-199,2010$.

[3] P. Colantoni "Color Space Transformations" 2004 [Online] http://faculty.kfupm.edu.sa/ics/lahouari/Teaching/colorspacetransf orm-1.0.pdf retrieved 24th October, 2018.

[4] O. Skrede "Color Images, Color Space and Color Image Processing". Lecture note in Digital Image Processing, Department of Informatics, The Faculty of Mathematics and Natural Sciences University of Oslo, 8th March 2017.

[5] K.N. Plataniotis and A.N. Venetsanopoulos. Color Image Processing and Applications. Springer-Verlag, Newyork.

[6] S. Sejpal and N. shah "Comparative Performance Analysis of Secured LWT-SVD Based Color Image Watermarking Technique in YUV, YIQ and YCbCr Color Spaces" In International Journal of Computer Applications (0975 - 8887) Vol. 147, no.7, pp. 3440, August 2016.

[7] P.R. Reddy, M.V.N.K. Prasad and D. S. Rao, "Robust Digital Watermarking of Color Images under Noise attacks", International Journal of Recent Trends in Engineering, Vol 1, No. 1, May 2009.

[8] B. L. Gunjal, "Wavelet Based Color Image Watermarking Scheme Giving High Robustness And Exact Corelation", International Journal Of Emerging Trends In Engineering And Technology (Ijetet) Vol. I No. 1, 2011.

[9] M. P Mitrea, F.J Preteux and A. Vlad "Robust Watermarking Method for Colour Still Image Databases" 2002. [online]

http://citeseerx.ist.psu.edu/viewdoc/download?doi=10.1.1.500.314

2\&rep=rep1\&type=pdf retrieved 24th December, 2018.

[10] I. Cox, et. al., "Secure Spread Spectrum Watermarking for Multimedia" in IEEE Transactions on Image Processing, vol.6, no.12,pp. 1673-1687, 1997.

[11] J. Ó Ruanaidh, and T. Pun, "Rotation, scale and translation invariant digital image watermarking", in Signal Processing, 66, 3, pp. 303-317, May 1998.

[12] Noel C. F. Codella, David Gutman, M. Emre Celebi, Brian Helba, Michael A. Marchetti, Stephen W. Dusza, Aadi Kalloo, Konstantinos Liopyris, Nabin Mishra, Harald Kittler, Allan Halpern: "Skin Lesion Analysis Toward Melanoma Detection: A Challenge at the 2017 International Symposium on Biomedical Imaging (ISBI), Hosted by the International Skin Imaging Collaboration (ISIC)", 2017; arXiv:1710.05006.

[13] Tschandl, P., Rosendahl, C. \& Kittler, H. The HAM10000 dataset, a large collection of multi-source dermatoscopic images of common pigmented skin lesions. Sci. Data 5, 180161 doi:10.1038/sdata.2018.161, 2018.

[14] R. Eswaraiah and E.S Reddy. "Robust medical image watermarking technique for accurate detection of tampers inside region of interest and recovering original region of interest" In IET image Process, vol. 9, no. 8 , pp. 615 - 625, Doi:10.1049/ietipr.2014.0986, 2015.

[15] R. Li, S. Xu, B. Rong and H. Yang "Host Cancellationbased spread spectrum watermarking for audio anti-piracy over Internet" In Security and Communications networks, vol. 9, pp. 4691 - 4702, October, 2016.

[16] H.S Malvar and D.A Florencio. "Improved Spread Spectrum: a new modulation technique for robust watermarking" In IEEE Transactions on Signal Processing, Vol. 51, no.4, pp. 898 -905, 2003.

[17] P. Eze, P. Udaya., and R. Evans. "Medical Image Watermark and Tamper Detection Using Constant Correlation Spread Spectrum Watermarking". World Academy of Science, Engineering and Technology, International Science Index 135, International Journal of Computer, Electrical, Automation, Control and Information Engineering, vol. 12, no. 3, pp. 107 - 114, 2018. 